\title{
marges Marges
}

revue d'art contemporain Revue d'art contemporain

12 | 2011

Exposition sans artiste(s)

\section{La programmation artistique en milieu scolaire : un cas avéré d'instrumentalisation de l'art?}

Artistic programming in a primary or secondary education context: is it a demonstrated case of an unfair exploitation of art?

\section{Géraldine Miquelot}

\section{OpenEdition}

\section{Journals}

Édition électronique

URL : http://journals.openedition.org/marges/410

DOI : $10.4000 /$ marges. 410

ISSN : 2416-8742

\section{Éditeur}

Presses universitaires de Vincennes

Édition imprimée

Date de publication : 15 avril 2011

Pagination : 64-77

ISBN : 978-2-84292-271-9

ISSN : $1767-7114$

\section{Référence électronique}

Géraldine Miquelot, «La programmation artistique en milieu scolaire : un cas avéré

d'instrumentalisation de l'art? », Marges [En ligne], 12 | 2011, mis en ligne le 15 avril 2011, consulté le 05 mai 2019. URL : http://journals.openedition.org/marges/410 ; DOI : 10.4000/marges.410 


\section{La programmation artistique en milieu scolaire: un cas avéré d'instrumentalisation de l'art?}

Les collections publiques d'art contemporain sont des agents du monde de l'art au même titre que les musées ou les centres d'art contemporain. Elles ont pour mission la diffusion d'œuvres sur un territoire donné. Cette diffusion passe par l'organisation d'expositions dans des lieux non artistiques tels que des écoles, hôpitaux, magasins, espaces publics, etc. La plupart de ces projets sont pilotés par les équipes des collections, notamment les personnes en charge des actions pédagogiques (responsables des publics, chargés d'action éducative, chargés de médiation...). À ces projets s'ajoutent des prêts d'œuvres, demandés par d'autres acteurs du monde de l'art dans le cadre d'expositions temporaires dans des institutions artistiques (musées, centres d'art). Les «projets» sont ainsi différenciés de ces prêts en raison de l'implication des équipes pédagogiques. La diffusion des collections est faite, en premier lieu, en direction des établissements scolaires, au titre de la mission de «partenariat avec le monde scolaire et 
universitaire » confiée aux structures d'art contemporain soutenues par le ministère de la Culture/1. Les établissements scolaires sont également le lieu d'autres projets artistiques à visée pédagogique mis en place par des structures artistiques: résidences d'artistes et productions d'œuvres. Le lieu de ces actions laisse penser qu'il s'agit, en soi, d'actions qui ne peuvent être envisagées comme de véritables programmations artistiques. Pourtant, le recours aux équipes et aux outils professionnels inscrit ces projets dans le monde de l'art. Leur rôle dans ce monde, apparemment proche de zéro, conduit à penser que les projets pédagogiques «instrumentalisent/2 » les artistes et les œuvres au profit de l'éducation artistique. En fait, il semble que cette instrumentalisation ne soit que rarement dénoncée comme telle - alors que l'accusation d'instrumentalisation est très fréquente dans les situations où les enjeux artistiques rencontrent des enjeux politiques, économiques, etc. Il semble alors peu pertinent de chercher à valider ou invalider cette hypothèse. Nous tenterons plutôt, à travers des études de cas, de «mesurer » leur degré d'instrumentalisation au sens large: le poids respectif de la fin pédagogique et de la fin artistique dans ces projets.

\section{Les collections publiques}

Les collections publiques d'art contemporain ont été créées, pour la plupart, au début des années 1980, alors que les mouvements de décentralisation politique (amorcés dans les années 1960) rencontraient le développement des politiques culturelles. On a parlé à cette période du renouveau de la «décentralisation culturelle», faisant référence à la politique d'équipement en Maisons de la culture lancée par André Malraux. Différentes sortes de collections ont été créées à cette occasion, toutes partageant la même mission: constituer une collection d'art contemporain, un «fonds» plus précisément, attaché à un territoire. Cela ne signifiait pas systématiquement que les achats étaient réservés à des artistes locaux, mais que la collection devait être conservée et diffusée en priorité sur ledit territoire/3. Ces fonds ont été créés initialement sans lieux d'exposition ("sans murs»), afin de favoriser leur diffusion dans d'autres lieux et «irriguer » ainsi tout leur territoire d'appartenance. II fallait surtout les différencier des musées des beaux-arts ou des musées d'art contemporain qui, eux, se caractérisent par un espace architectural plus ou moins imposant, souvent implanté dans une grande ville et surtout identifiable. L'idée d'un fonds assimilé non pas à un lieu mais à un territoire était également associée à celle d'une diffusion dans des lieux non artistiques, sous la forme possible d'expositions itinérantes. Au début des années 1980, il y avait
/1 1 Ministère de la Culture et de la Communication, Charte des missions de service public pour les institutions d'art contemporain, 27 novembre 2000 [http://www.culture.gouv. $\mathrm{fr} /$ culture/actualites/ politique/chartes/ chartes-dap.html] consulté le 15 décembre 2009, p. 5.

/2 Instrumentaliser: considérer quelque chose comme un instrument, purement utilitaire. Comme un moyen plutôt qu'une fin.

/3 Pour la conservation sur le territoire, c'est possible dans le cas des régions mais les collections départementales et municipales ne sont pas systématiquement ni intégralement conservées sur leur territoire de rattachement. 
/4 "Circulaire du 23 juin 1982 relative à la création des Fonds régionaux d'acquisition d'œuvres d'art contemporain. (non publiée au journal officiel) ", accessible au http://www.paca.culture. gouv.fr/ressources/ text_lois/lois/C-23-06-82AP-FRAC.pdf (consulté le 7 février 2010).

/5 Seule exception: le FRAC Picardie fait partie de l'Office culturel régional de Picardie, opérateur des actions culturelles sur le territoire régional (Roxana Azimi, «Frac Picardie, Mue en vue ", Le Journal des Arts, $\mathrm{n}^{\circ} 302$, du 2 au 14 mai 2009, p. 9). Le FRAC Corse, créé sous la forme associative en 1986 a été intégré au Conseil Régional en 1998. Il a tout de même une direction artistique, et est membre du réseau Platform.

/6 À ma connaissance il n'y en a que deux qui n'ont pas de lieu d'exposition propre: le FRAC Corse et le FRAC Réunion, mais ces derniers ont des partenaires privilégiés pour l'organisation d'expositions. moins de lieux d'art contemporain qu'aujourd'hui et la diffusion des œuvres, nécessairement, devait « déborder » dans les lieux non artistiques mais ouverts au public tels que les écoles, hôpitaux, etc.

Depuis, les centres d'art contemporain et autres lieux d'expositions se sont multipliés sur l'ensemble du territoire français. À la faveur de cette multiplication, de nombreuses collections publiques se sont dotées de lieux d'exposition. Même si ces espaces ne sont pas consacrés exclusivement à la collection à laquelle ils sont rattachés, ils ont fortement contribué à améliorer leur visibilité et leur identité. Les différents types de fonds d'art contemporain se distinguent par leur territoire d'inscription bien sûr, mais aussi par leur statut juridique, leurs liens avec un ou plusieurs lieux d'exposition et leurs partenaires publics.

\section{Les Fonds régionaux d'art contemporain ou «FRAC »}

Les Fonds régionaux d'art contemporain ou «FRAC» sont les collections publiques les plus connues et les plus homogènes, car issues d'une initiative ministérielle de 1982/4. Chaque région française dispose d'un FRAC. La circulaire relative à leur création précise que ces fonds doivent avoir un financement croisé entre, au minimum, l'État et la Région, et être gérés par des associations. Aujourd'hui, la plupart des FRAC ont encore le statut d'association (loi de 1901), ce qui leur assure une relative flexibilité financière. Leur statut de «personne morale» permet leur adhésion à des associations de structures comme Platform, association nationale qui rassemble aujourd'hui vingt-et-un FRAC, ou des associations régionales d'art contemporain du type Tram en île-de-France, ACB en Bretagne, etc./5. Leur Conseil d'administration regroupe des représentants des collectivités locales et de la DRAC (Ministère de la Culture et de la Communication), et leurs comités techniques, chargés de proposer des acquisitions, sont composés de professionnels de l'art contemporain.

Créés sans murs, les FRAC disposent aujourd'hui presque tous d'un espace d'exposition propre ou sont sur le point d'en être équipés/6. Depuis les années 1990, il leur est devenu nécessaire de s'aligner sur le vaste mouvement d'équipement du territoire français en lieux d'expositions. On parle alors des FRAC de « deuxième génération ». En Îlede-France, le FRAC et le centre d'art contemporain Le Plateau, depuis sa création en 2002, sont associés à la demande du Conseil Régional. Même équipés de lieux d'exposition, les FRAC continuent la diffusion de leur collection dans divers lieux non artistiques, parmi lesquels les établissements scolaires sont très nombreux.

Les financements des FRAC sont variés, mais la majorité bénéficie exclusivement de fonds publics. Le partenariat financier entre État et Région (exigé par l'État) est présent partout, parfois associé à un 
financement de la Ville et/ou du Conseil Général. Ces modes de financement déterminent directement les groupes scolaires vers qui se tournent les équipes pédagogiques des $\mathrm{FRAC} / 7$.

Du point de vue du projet artistique, les FRAC ont un directeur qui donne les grandes orientations de la programmation. Le directeur peut être commissaire des expositions ou inviter d'autres commissaires, voire des artistes, à travailler à partir de la collection. Les FRAC sont donc comparables aux centres d'art par leur programmation d'exposition dans ou hors de leurs murs. Ils s'inscrivent ainsi dans le paysage de l'art contemporain par leur double fonction: un soutien aux artistes par des achats, des expositions et des productions, parfois des éditions; une action vers les publics par la médiation proposée sur leurs expositions et par la diffusion de leur collection. Ceci, associé à leur adhésion à une ou plusieurs associations de lieux d'art contemporain, légitime leurs choix en matière de programmation artistique.

\section{Les collections départementales}

Dans les mêmes années qui ont vu la création des FRAC, certains départements se sont dotés de collections d'art contemporain. Appelées «FDAC» par comparaison avec les FRAC, ces collections en sont en fait assez différentes. Initiées par les Conseils Généraux, elles ne sont pas systématiques (tous les départements n'en ont pas) ni homogènes d'un département à l'autre.

La Collection publique d'art contemporain du Conseil Général de Seine-Saint-Denis par exemple a été créée en 1986. Elle est intégrée à la Direction de la Culture, du Patrimoine, du Sport et des Loisirs, puis au Service de la Culture, enfin au Bureau des Arts visuels et du Cinéma. La Collection est donc l'un des services offerts par le Conseil Général aux habitants de la Seine-Saint-Denis. À la différence des FRAC, elle n'a pas de direction artistique propre. La collection de la Seine-SaintDenis est diffusée sur le territoire séquano-dionysien de diverses manières, le plus souvent en partenariat avec des structures locales. Cette diffusion peut se faire sous la forme d'expositions en partenariat avec des lieux d'exposition/8, auquel cas le Conseil Général est considéré comme co-initiateur du projet artistique. Ce type de projet en lieux identifiés comme lieux d'expositions artistiques s'inscrit dans le monde de l'art contemporain et définit de ce fait la Collection comme agent de ce monde. En revanche, cette légitimation est moins évidente dans le cas où la Collection est diffusée en milieu scolaire ou en partenariat avec un établissement scolaire - et ce, même en présence d'une structure culturelle partenaire. De même, la Collection étant intégrée à une administration territoriale, elle ne peut adhérer à
/ 7 Le cas du FRAC Languedoc-Roussillon par exemple, est significatif. Ce FRAC est financé par la DRAC (Ministère de la Culture et de la Communication) et la Région LanguedocRoussillon. L'équipe des publics (deux personnes) répond donc aux sollicitations d'établissements municipaux ou départementaux, mais a concentré ses actions volontaristes en directions des lycées, établissements gérés par la Région.

/8 C'est le cas pour la récente exposition «Ce que j'ai sous les yeux, exposition de la Collection d'art contemporain du Conseil Général», Musée d'Art et d'Histoire, Saint-Denis, du 3 octobre 2009 au 25 janvier 2010. 
/9 Seul le chantier du musée a bénéficié de financements de la Région et de la DRAC.

/10 D'ailleurs le Mac/Val est membre du réseau Tram et ses directeurs sont reconnus comme acteurs du monde de l'art si l'on se fie à leur invitation comme commissaires de la Nuit Blanche 2009.

/11 On peut donc faire I'hypothèse que le FMAC est né dans la même dynamique que les FRAC et FDAC. des associations de structures artistiques. Il y a donc, pour cette Collection, moins de moyens pour lui attribuer une légitimité artistique, et ce même si elle travaille avec des structures légitimes.

À titre de comparaison, la collection du Val-de-Marne (fondée dans les années 1980) a d'abord fonctionné sur le même mode que la Collection du Conseil Général de la Seine-Saint-Denis: diffusion dans des lieux ouverts au public dans le département, partenariats avec les établissements (scolaires et autres), partenariats pédagogiques avec des structures culturelles, prêts d'œuvres pour des expositions temporaires. Le Fonds départemental d'art contemporain (FDAC) a donné lieu à un projet de musée, dont la localisation (Vitry) a été choisie en 1990 donc très rapidement après sa fondation. Le FDAC est devenu «musée» en 1999, sous le contrôle scientifique de l'État (aujourd'hui labellisé Musée de France), alors que le musée en luimême, le Mac/Val a ouvert en 2005. Aujourd'hui, le Mac/Val ne fonctionne qu'avec des financements du Conseil Général (sauf aides au projet ponctuelles), et une aide de la Direction des Musées de France pour son action scientifique/9. Du point de vue scientifique justement, disons «artistique», le Mac/Val fonctionne avec une double direction artistique (une conservatrice en chef et un chargé des expositions), ce qui le place d'emblée du côté des acteurs du monde de l'art/10.

Contrairement à ce qui s'est passé pour les FRAC, l'ouverture du musée du Mac/Val a stoppé net sa diffusion sur le territoire. En effet, la politique de «diffusion» de la collection a été inversée: les œuvres ne sont plus exposées dans le territoire (sauf prêts pour expositions temporaires, dans des lieux artistiques) mais le musée fait venir les groupes sur place. Cette politique centripète fait du Mac/Val un lieu de destination privilégiée des groupes scolaires - notamment les collèges du Val-de-Marne.

\section{Le Fonds municipal d'art contemporain de la Ville de Paris}

Le Fonds municipal d'art contemporain de la Ville de Paris (FMAC) est la seule collection municipale d'art contemporain sans murs. Issue des Collections municipales fondées en 1816, elle a été divisée en 1987 entre un ensemble d'œuvres modernes confiées au Musée du Petit Palais (Musée des Beaux-Arts de la Ville de Paris) et un ensemble d'œuvres contemporaines rassemblées sous l'acronyme FMAC/11. Le FMAC fait partie du Département de l'Art dans la Ville, au sein de la Sous-direction de la Création Artistique, elle-même au sein de la Direction des Affaires Culturelles. C'est donc un service de la Ville.

Destinées à circuler dans les locaux municipaux, les œuvres ont beaucoup été montrées dans les bureaux bien que certaines soient occasionnellement prêtées pour des expositions temporaires en structu- 
res artistiques. Ce n'est que récemment que l'équipe en charge de la collection a développé son action vers le public. Cette action s'est manifestée par l'organisation d'expositions en structures culturelles: au musée du Petit Palais par exemple, où il s'agissait de faire dialoguer des œuvres contemporaines et des œuvres classiques/12; à la Foire Internationale d'Art Contemporain (FIAC), où le FMAC montre ses dernières acquisitions, chaque année, depuis 2003. Ces deux types d'exposition inscrivent le FMAC dans des missions artistiques: commissariat d'exposition, démonstration de son soutien aux artistes. Plus récemment, le FMAC a lancé une vaste opération de prêts d'œuvres en établissements scolaires, associés à des actions de médiation adaptées à chaque œuvre: "Le FMAC à l'école», qui inaugure en quelque sorte, une politique volontariste et visible d'action pédagogique.

\section{Les projets mis en place}

On a vu que, parmi les collections publiques, certaines sont plus facilement identifiées comme structures d'art contemporain que d'autres. Cela n'empêche pas que toutes adoptent pour leur action pédagogique vers les groupes scolaires, des formes d'action qui reprennent (pour ne pas dire qui «singent») les formes d'une programmation artistique. Si le but de ces actions pédagogiques est de faire découvrir l'art contemporain, il est logique qu'elles en reprennent les formes. Mais comme leur rôle dans la carrière de l'artiste est inégal, voire anecdotique, on pense inévitablement à l'instrumentalisation des œuvres, voire des artistes.

\section{L'exposition d'œuvres de la collection}

Une collection, dont les œuvres ne sont, nécessairement, pas toutes exposées, constitue en quelque sorte une « réserve disponible ». Il est alors très simple de monter une exposition en fonction d'un projet pédagogique. Alors que pour un centre d'art, les expositions (manifestations artistiques) sont un point de départ courant pour concevoir les actions pédagogiques destinées aux groupes scolaires. Une exposition mise en place sur la base d'un projet pédagogique peut apparaître totalement déconnectée du monde de l'art contemporain. C'est ce degré de déconnexion qu'on va analyser ici, en observant comment les projets peuvent osciller entre «l'artistique » et «le pédagogique». Le premier cas analysé ici est un projet organisé par le FRAC Île-deFrance en 2007/2008, qui consistait en l'exposition d'œuvres de la collection dans cinq lycées, sur une période de deux mois. Pour cela, le FRAC s'est associé à cinq structures culturelles (membres du réseau Tram), à chaque fois à proximité d'un lycée, pour coordonner le projet.
/12 «Intrusions, le Fonds municipal d'art contemporain au Petit Palais », Petit Palais, Musée des Beaux-arts de la Ville de Paris, du 6 octobre 2007 au 8 janvier 2008 . 
/13 Respectivement vice-président du Conseil Régional d'île-de-France, chargé de la Culture et des TIC, et directeur du FRAC Île-de-France.

/14 D'ailleurs, le projet était surnommé " 5 lycées» par l'équipe du FRAC et ses partenaires culturels.

/15 Brochure de l'exposition au lycée Théodore Monod.
Si l'on considère le FRAC comme une structure culturelle légitime, le projet était donc fondé sur des partenariats mettant en scène dans chaque cas un acteur culturel général, un acteur culturel «relais» et un établissement scolaire.

Du point de vue du FRAC, le projet était clairement pédagogique tout en bénéficiant d'une grande visibilité : brochure publiée sur chaque lycée (dans la charte graphique du FRAC), avec un mot d'introduction de Francis Parny et Xavier Franceschi/13, carton d'invitation protocolaire, vernissages dans chaque lycée, et publication pour l'une des expositions (dans la collection éditoriale lancée par le FRAC quelque temps auparavant). Cela dit, le projet était coordonné par la responsable du service des publics du FRAC, il portait le titre «Jean-Pierre Timbaud, Galilée, Antoine de Saint-Exupéry, Jean Jaurès, Théodore Monod », soit la liste des noms des cinq lycées concernés/14. De plus chaque brochure précisait que les expositions étaient ouvertes en priorité aux lycéens et aux enseignants - ce qui à la fois affirme la vocation pédagogique du projet, et justifie que les lycées n'aient pas été plus ouverts au public à ce moment-là. Le double niveau de partenariat (FRAC-structures culturelles relais puis structures-lycées) a donné lieu à cinq projets différents sur le plan de l'équilibre artistique/pédagogique. Finalement, seules les informations diffusées par le FRAC étaient homogènes.

L'une de ces expositions a eu lieu dans le lycée Théodore Monod à Noisy-le-Sec, coordonnée par La Galerie, centre d'art contemporain de Noisy-le-Sec. Pour ce cas-là, la directrice de La Galerie a décidé d'intégrer ce projet d'exposition pédagogique à son projet artistique. Elle a donc choisi les œuvres de telle sorte qu'elles résonnent avec l'exposition du centre d'art pendant cette période. L'exposition dans le lycée était présentée comme « une extension/15 » de celle du centre d'art (ce qui entrait quelque peu en contradiction avec la mention du FRAC sur l'ouverture des expositions), la directrice de La Galerie étant indiquée comme commissaire des deux expositions. L'exposition du lycée était également présentée dans la brochure de l'exposition "Visions nocturnes» en cours à La Galerie à ce moment, et portait le même titre. Tout l'appareil de l'exposition artistique était en place (communication, questions d'accrochage, commissariat d'exposition, etc.) : il s'agissait d'un projet affirmé comme artistique avant d'être pédagogique. Cela n'a pas empêché - et même, cela a permis - l'organisation d'actions éducatives très denses auprès des lycéens, à l'image de la politique éducative générale du centre d'art La Galerie. Un autre cas d'exposition pédagogique est fondé également sur un partenariat à trois interlocuteurs: le projet «Une œuvre à soi », initié par le Conseil Général de la Seine-Saint-Denis, s'étend sur tout le territoire du département en s'appuyant sur des structures cultu- 
relles relais. Le cas étudié ici est celui mené en partenariat avec la Maison Populaire, association pluridisciplinaire de Montreuil, soutenue (financièrement) par le Conseil Général pour sa programmation culturelle, et qui comprend entre autres un lieu d'exposition d'art contemporain. Le programme «Une œuvre à soi » se déroule sous forme de cycles annuels de trois accrochages et associe des enseignants volontaires (d'une dizaine d'établissements scolaires). Ces derniers choisissent les thèmes du cycle, à partir de quoi les œuvres sont sélectionnées par la responsable de la médiation à la Maison Populaire et la responsable de la Collection au Conseil Général. Trois fois par an, durant quelques semaines, les œuvres sont exposées dans le salon de la Maison Populaire (un espace d'accueil, distinct du centre d'art), et des «ateliers du regard » sont organisés avec les classes des enseignants partenaires du projet.

La vocation pédagogique de ce projet est bien plus importante que sa vocation artistique: les œuvres sont choisies pour leur médium et pour répondre aux thèmes de travail des enseignants; le lieu est un lieu de vie et non l'espace d'exposition de la Maison Populaire; l'exposition ne bénéficie d'aucun outil professionnel artistique, à l'exception de la convention de prêt des œuvres et du transport: ni carton ni vernissage, les artistes ne sont guère prévenus de l'exposition de leurs œuvres - tout juste sont-ils, s'il habitent l'île-de-France, invités à venir voir l'accrochage. Outre cette dimension pédagogique prédominante, la différence avec le cas du FRAC est le statut de l'un des partenaires: dans ce projet, le Conseil Général est à la fois partenaire financier de la Maison Populaire pour sa programmation artistique et partenaire artistique par le choix et le prêt des œuvres.

Le dernier cas est celui du «FMAC à l'école»: un projet d'exposition réalisé «en interne » car les partenaires en jeu, s'ils sont a priori très nombreux (29 écoles sont concernées), dépendent tous de la même administration territoriale, la Ville de Paris. Le FMAC a lancé cette action en 2009 auprès des écoles du $11^{\mathrm{e}}$ arrondissement, auxquelles des écoles d'autres quartiers se sont ajoutées au cours de l'élaboration du projet. La mise en place des partenariats a été très protocolaire. Le FMAC s'est chargé des relations «diplomatiques» : contact avec les maires d'arrondissements et liens entre les deux directions concernées (Direction des Affaires Culturelles et Direction des Affaires Scolaires), tandis que chaque mairie d'arrondissement impliquée a contacté les directeurs d'établissements et les enseignants d'arts plastiques, qu'ils soient employés de la Ville ou de l'État.

C'est la responsable actuelle du FMAC qui a présélectionné des œuvres pour ce projet, puis invité les enseignants et les directeurs d'établissements à choisir les œuvres pour leur école. Chacune des 29 
/16 En janvier 2010, il était question de publier une «brochure/album» qui comporterait les œuvres du projet, leur notice, ainsi que les actions de médiation mises en place pour chacune d'elles.

/17 L'usage du terme «restitution» connote une exposition issue d'une année d'ateliers. écoles accueille une œuvre ou un ensemble d'œuvres, exposée(s) de manière à ce que le maximum de personnes, y compris les parents d'élèves, les voient, donc souvent dans l'entrée de l'établissement. La responsable du FMAC parle de véritable «exposition » pour ce projet, mais c'est une exposition comparable non aux prêts d'œuvres pour expositions temporaires, mais aux prêts d'œuvres aux élus de la Ville: ce ne sont pas des conventions de prêt mais des formulaires de choix d'œuvres qui sont signés. Dans la mise en place, c'est le FMAC qui gère la majeure partie du projet: transport, soclage ou accrochage, documentation, médiation. Les artistes sont parfois invités à rencontrer les élèves, mais pas systématiquement. En revanche, tous sont prévenus de l'exposition de leur œuvre dans l'établissement scolaire.

Il s'agit donc d'une action qui utilise fortement les outils du monde de l'art professionnel (de la prise de décision initiale à la gestion technique), mais la communication faite sur l'événement, et le projet de publication, insistent sur la teneur pédagogique de l'événement/16. D'ailleurs, dans les écoles où l'œuvre n'a pas été exposée dans l'entrée mais à l'intérieur de l'école, une photographie de l'œuvre en situation a été réalisée pour être montrée à l'entrée, afin que les parents d'élèves puissent profiter tout de même de l'exposition. On imagine très mal ce genre de substitution à l'entrée d'un musée.

\section{Les résidences d'artistes en collège}

Dans la poursuite de son action culturelle vers les établissements scolaires, le Conseil Général de la Seine-Saint-Denis propose depuis quelques années des résidences d'artistes en collège. L'un de ces programmes de résidence est intitulé «In situ ». Les objectifs du projet ne sont pas formulés en termes artistiques: pas d'exposition en vue, pas de perspective d'achat d'œuvre ni même d'objectif de production. Pourtant, les artistes sont choisis en fonction de leur projet artistique, et de leur activité professionnelle (diffusion en milieu artistique légitime, recommandation par des professionnels...). Cette année, onze artistes, dont deux plasticiennes, sont concernés, répartis dans dix collèges (l'un des artistes étant en «résidence itinérante» dans les dix collèges).

Le contrat passé entre l'artiste et le Conseil Général impose, sur les neuf à dix mois de la résidence, un minimum de 40 heures de "projet culturel», c'est-à-dire d'action pensée en lien avec une classe et plusieurs enseignants, comportant: des temps d'atelier, des visites culturelles et une restitution/17 finale. Bien entendu, les artistes peuvent travailler avec plus de classes s'ils le souhaitent, et dépasser les 40 heures de projet. Une bourse est octroyée à l'artiste, et un budget spécifique est réservé pour les projets culturels. C'est pour la gestion 
de ce dernier budget qu'interviennent d'autres structures culturelles. Pour chaque résidence, une structure culturelle relais est en effet désignée comme opératrice des projets culturels de l'artiste. Le rôle et l'importance de cette structure dans la mise en place et la conduite des projets varie selon les cas, de simple opérateur financier à coorganisateur des projets culturels. L'implication de l'artiste dans ces projets culturels varie également.

Le but de ces résidences s'inscrit très clairement dans une démarche pédagogique et même scolaire. Ce programme, ainsi que toutes les actions culturelles en collèges, est présenté par le Conseil Général comme faisant suite à une étude/18 démontrant que les projets culturels, dans certaines conditions, développent des capacités (d'apprentissage, notamment) chez les élèves. De ce fait, les artistes en résidence sont en contact à la fois avec une personne chargée de mission éducative et une personne chargée de mission culturelle au sein du Conseil Général. De plus, la classe référente de chaque résidence a été "créée», au cours de l'année scolaire précédente, spécifiquement pour cette résidence, afin de répartir chez les élèves les «bienfaits» du projet.

Une expérience différente de résidence d'artiste en lycée a été menée par le FRAC Île-de-France en 2006/2007, au lycée Galilée de CergySaint-Christophe. Le FRAC et le lycée entretenaient des relations étroites par l'organisation fréquente d'expositions pédagogiques, qui avaient abouti à l'ouverture d'une galerie/19. Ce partenariat, établi sur le long terme, a donné lieu à la résidence d'une artiste (représentée dans la collection) dans le lycée. Durant toute la durée de sa résidence, elle a produit des œuvres, rencontré les lycéens, organisé avec eux des expositions avec d'autres œuvres de la collection. Une grande partie du projet a donc bénéficié d'une légitimité artistique. Le FRAC était en fait initiateur de la résidence et a maintenu son caractère " artistique » par divers moyens: l'une des expositions de la galerie du lycée a été intégrée à une série de visites de lieux du réseau Tram, dont on a vu le rôle «légitimant». Autre exemple: certaines œuvres produites par l'artiste durant sa résidence ont été acquises dans la collection.

\section{Convergences et divergences avec le monde de l'art}

Tous ces projets ont en commun d'être initiés par des collections publiques d'art contemporain, mais celles-ci agissent soit comme structures artistiques légitimes, soit en tant que collectivités locales. Ces projets d'expositions et de résidences mobilisent en grande partie les outils, les compétences et le vocabulaire d'événements artistiques. Il s'agit d'«expositions» réalisées parfois par des «commissaires
/18 L'étude dont il est question est mentionnée en plusieurs endroits du site Internet www.seinesaint-denis.fr: «Ambition "Réussite scolaire pour tous”. Les démarches éducatives sous la loupe des sociologues ", Laboratoire ESCOL-ESSI (Paris 8), Conseil Général de la Seine-Saint-Denis, novembre 2006.

/19 Labellisée Espace de relation à l'œuvre d'art ou «EROA». 
d'exposition"; ;'emballage, les transports et l'accrochage sont faits par les professionnels des lieux d'art contemporain; les exigences de conservation qui jouent sur l'accrochage sont les mêmes que pour une exposition artistique. Lorsqu'il y a communication, il est fréquent qu'elle soit faite dans la charte graphique de la structure culturelle relais, ou dans celle de la collection. On a vu également comment la structure initiatrice du projet ou la structure relais peuvent donner une légitimité artistique à un projet apparemment pédagogique, dans les cas du FRAC/La Galerie/Théodore Monod, ou FRAC/Galilée.

À l'exception de ces deux derniers cas, l'événement est présenté comme un événement pédagogique. Dans la présentation de leurs actions, les structures culturelles les classent dans les actions éducatives et non dans leur programmation artistique. De plus, tous les artistes dont les œuvres sont exposées ainsi ne sont pas systématiquement prévenus, alors que cet «oubli» est inconcevable pour un prêt d'œuvre en centre d'art. Et parmi ceux qui sont prévenus, rares sont ceux qui mettent en avant ces expositions dans leur biographie. Le seul cas où l'on ne peut se passer de la présence de l'artiste est celui de la résidence, où c'est la personne même de l'artiste qui est au cœur du projet. Dans le cas des résidences du Conseil Général de la Seine-Saint-Denis, le travail des artistes a motivé leur choix par le Conseil Général, mais ce même travail finit par devenir accessoire dans les cadres établis de la résidence.

Enfin, dans presque tous les cas, la collection s'appuie sur des partenaires locaux (encore plus locaux qu'elle-même, en quelque sorte) pour assurer, dans des modalités variables, le suivi du projet. Cette caractéristique territoriale place ces projets dans une situation de "proximité » qui met en contact des partenaires divers, alors que le monde de l'art contemporain fonctionne sur une logique territoriale inverse: des réseaux qui peuvent être très distendus géographiquement, mais homogènes dans leur composition. II n'est pas anodin que, dans le projet du FRAC au lycée de Cergy-Saint-Christophe, aucune structure culturelle n'intervenait entre le FRAC et le lycée.

Après cette étude de cas qui montre une pluralité de situations, on pourrait conclure que, d'une part, il y a bien instrumentalisation et que, d'autre part, cette dernière est plus ou moins évidente. C'est la nature de la participation d'une structure culturelle reconnue ou l'intervention d'une personne dont le rôle est indiscutable qui décide du caractère pédagogique ou artistique de ces projets. Dans le cas du partenariat entre le FRAC et La Galerie de Noisy-le-Sec, la directrice de La Galerie a maintenu un certain contrôle sur l'exposition pédagogique, de la même manière que le FRAC au lycée Galilée de Cergy-Saint-Christophe. Finalement, ces projets fonctionnent comme 
l'ensemble du monde de l'art: il suffit qu'un consensus se fasse sur la nature du projet pour qu'il soit admis comme artistique ou pédagogique. En l'absence de consensus, il faut qu'une personne relativement influente désire infléchir le projet dans une direction plutôt que dans une autre. Dans ce contexte, la nature des œuvres semble à ce point accessoire, que l'instrumentalisation ne fait plus de doute. Les œuvres ont beau être issues de collections publiques constituées d'œuvres elles-mêmes issues (en grande partie) du marché de l'art, la dominante artistique ou pédagogique des expositions en milieu scolaire est décidée par un tout autre jeu d'influences.

Pour que cette instrumentalisation soit à ce point admise (sinon ignorée) par l'ensemble des professionnels de l'art contemporain, quel intérêt peut-il y avoir pour eux, au-delà de rapports facilités avec leurs partenaires publics?

\section{De l'intérêt de l'«instrumentalisation »}

Pour une collection publique d'art contemporain indépendante des collectivités locales, il s'agit d'actions indispensables pour faire vivre la structure. Les équipes qui se consacrent à ces actions, même si elles agissent dans un monde parallèle au monde de l'art contemporain, élaborent des outils qui valorisent, par ailleurs, la structure: les cartons, catalogues, notices écrites à l'occasion des expositions pédagogiques alimentent les fonds de documentation sur les œuvres de la collection, et servent, à leur tour, le projet artistique de la structure gestionnaire de la collection. De plus, les mouvements d'une collection sont indispensables à la régie des œuvres: ce n'est que lors des déplacements d'œuvres qu'il est possible de remarquer et de résoudre les problèmes de conservation et de détérioration des œuvres elles-mêmes, ou de résoudre les problèmes d'emballage ou de soclage révélés par l'exposition. De même, du point de vue de la mission de service public de la collection publique, les interlocuteurs scolaires en jeu dans les expositions pédagogiques sont autant de partenaires potentiels pour des rencontres sur les expositions "artistiques », c'est-à-dire pour des projets pédagogiques issus du projet artistique de la collection. Enfin - et c'est peut-être l'argument le plus important - les projets pédagogiques sont une donnée importante que les partenaires publics demandent aux FRAC, et sur laquelle ils évaluent leurs besoins matériels, financiers, humains... Alors que la seule hausse de fréquentation d'une exposition n'implique pas nécessairement un accompagnement plus dense, l'augmentation du nombre de projets pédagogiques est un argument plus évident, pour les structures culturelles face à leurs tutelles, pour réclamer de nouveaux moyens. 
/ 20 Claude Bartolone, "Le Plan d'éducation artistique ", La Culture en Seine-Saint-Denis $n^{\circ} 7$, sans date, p. 1.
Pour une collection publique intégrée à une administration territoriale, les bénéfices sont moins liés aux partenaires financiers (quasi inexistants) qu'aux administrés, aux citoyens du territoire, c'est-à-dire au «public» de la collectivité, bien plus large que le public de ses actions artistiques. C'est la raison pour laquelle la légitimation artistique n'a finalement que peu d'intérêt pour ces projets. Les résidences d'artistes ou le projet «Une œuvre à soi » étant pilotés par des établissements scolaires et culturels, ils mobilisent deux services du Conseil Général, l'éducation et la culture. Cela place ces projets, notamment les résidences d'artistes, dans une fonction co-éducative tout à fait assumée. Bien entendu, lorsque les objectifs du projet «In situ» ont été présentés aux enseignants, il leur a été demandé de s'impliquer en étant capables de faire abstraction du programme scolaire. II n'en reste pas moins évident que ces projets de programmation artistique en milieu scolaire, en Seine-Saint-Denis, ont pour vocation une amélioration des rapports des élèves à l'apprentissage/20.

Lorsque je pose la question «À quoi sert l'art? » à des professionnels de l'art contemporain, la plupart commencent par dire que l'art «ne sert à rien» (ou disent «je pourrais répondre que ça ne sert à rien »), puis énumèrent des «effets » de l'art. C'est tout le paradoxe qui explique que l'instrumentalisation pédagogique des œuvres, voire des artistes, ne semble déranger personne: non seulement elle permet d'accéder à d'autres avantages, mais en plus elle se justifie par une pirouette rhétorique du type «l'art peut avoir des effets mais il ne doit pas être exploité».

Pour les structures reconnues comme artistiques, dont la mission première est de proposer une programmation artistique, ce serait contraire à leur vocation que d'affirmer utiliser l'art dans des objectifs pédagogiques ou éducatifs. C'est pourquoi les projets pédagogiques sont mis en avant dans des contextes très particuliers (dans la communication très locale ou lors d'échanges avec les partenaires financiers) et d'une manière qui ne fait aucun doute sur le partenariat avec les professionnels pédagogiques. Même si dans les faits, les structures culturelles sont souvent plus impliquées (en termes de moyens, de temps, d'équipe) dans ces projets que leurs partenaires scolaires, la simple présence de l'interlocuteur scolaire donne l'image d'une répartition idéale des rôles: l'artistique pour la structure culturelle, l'éducatif pour l'école.

Il est tout de même important de noter que ces projets présentent des avantages certains dans le monde de l'art, au-delà des relations facilitées entre structures culturelles et partenaires publics. Un artiste en résidence en collège se trouve équipé pour créer, produire et stocker des œuvres destinées au monde de l'art. Sur des crédits réservés 
aux actions pédagogiques, une structure culturelle peut s'équiper en matériel utile à la production d'expositions ultérieure. De la même manière, les relations entre un artiste et une structure artistique seront facilitées si, à la faveur d'un projet pédagogique externe au projet artistique, la structure peut lui proposer un revenu: cela permettra à la structure d'être plus exigeante vis-à-vis de l'artiste.

L'affirmation d'une «fonction » de l'art est plus facilement admise dans le cas d'une administration territoriale: c'est son rôle de proposer des services dans différents domaines de la vie quotidienne, y compris la culture et l'éducation, donc pourquoi ne pas mêler les deux?

$\mathrm{Au}$ vu de l'organisation du programme «In situ», on pourrait se poser la question d'une autre sorte d'instrumentalisation: celle des structures culturelles. Puisque c'est le Conseil Général qui choisit les artistes, les collèges, et les structures partenaires, il devient financeur et programmateur du projet, tandis que les structures culturelles deviennent de simples opérateurs; alors que leur rôle classique est de programmer des projets sur lesquels le Conseil Général est cofinanceur. En somme, c'est comme si les partenaires devenaient programmateurs et les programmateurs, partenaires. C'est peutêtre un moyen pour le Conseil Général de contrôler plus étroitement les actions pédagogiques des structures culturelles qu'il soutient, mais en tout cas cela confirme la confusion des rôles des différents acteurs, perceptible dans l'ensemble du monde de l'art contemporain et même, dans l'ensemble des mondes de l'art.

\section{Géraldine Miquelot}

